\title{
Leading to Distraction: Driver distraction, lead car, and road environment
}

\author{
Georgios K. Kountouriotis $^{1 *} \quad$ Natasha Merat $^{2}$ \\ ${ }^{1}$ Department of Psychology, Manchester Metropolitan University \\ ${ }^{2}$ Institute for Transport Studies, University of Leeds \\ ${ }^{*}$ Corresponding author: g.kountouriotis @mmu.ac .uk \\ This article may not exactly replicate the final version published in the Accident Analysis \& Prevention. It is not the \\ copy of record. \\ (C)2016. This manuscript version is made available under the CC-BY-NC-ND 4.0 license \\ http://creativecommons .org/licenses/by-nc-nd/4.0/
}

\begin{abstract}
Driver distraction is strongly associated with crashes and near-misses, and despite the attention this topic has received in recent years, the effect of different types of distracting task on driving performance remains unclear. In the case of non-visual distractions, such as talking on the phone or other engaging verbal tasks that do not require a visual input, a common finding is reduced lateral variability in steering and gaze patterns where participants concentrate their gaze towards the centre of the road and their steering control is less variable. In the experiments presented here, we examined whether this finding is more pronounced in the presence of a lead car (which may provide a focus point for gaze) and whether the behaviour of the lead car has any influence on the driver's steering control. In addition, both visual and non-visual distraction tasks were used, and their effect on different road environments (straight and curved roadways) was assessed. Visual distraction was found to increase variability in both gaze patterns and steering control, non-visual distraction reduced gaze and steering variability in conditions without a lead car; in the conditions where a lead car was present there was no significant difference from baseline. The lateral behaviour of the lead car did not have an effect on steering performance, a finding which indicates that a lead car may not necessarily be used as an information point. Finally, the effects of driver distraction were different for straight and curved roadways, indicating a stronger influence of the road environment in steering than previously thought.
\end{abstract}

Keywords: Driving, Distraction, Lead Car, Gaze

\section{Introduction}

Driving a car is a task which involves the acquisition of many complex skills (Groeger, 2000). In recent years, the introduction of in-vehicle and nomadic technologies such as smart phones and navigation systems has meant that driving is now often accompanied by other, competing, tasks. It is well-established that engagement in such 'secondary tasks' can compromise driving safety, with naturalistic studies claiming that approximately $78 \%$ of all crashes and near misses are related to driver inattention and distraction (Klauer et al., 2006).

As argued by information processing models such as the Multiple Resource Theory (Wickens, 2002) or the Working Memory Model (Baddeley, 1992), how distraction affects drivers appears to depend largely upon the type of secondary tasks used, with the main distinction being between distracting tasks that rely on presentation of visual information (visual distraction) and therefore take drivers' eyes away from the road, and distraction tasks that have no visual component, or at least do not require drivers' eyes to be taken away from the 
road (non-visual distraction) ${ }^{1}$.

Visual distraction has been shown to increase the vehicle's lateral deviation from the centre of the lane (Engström et al., 2005; Santos, Merat, Mouta, Brookhuis, \& de Waard, 2005; Liang \& Lee, 2010) and also increase the deviation of eye gaze (Victor, Harbluk, \& Engström, 2005; Reyes \& Lee, 2008). This increase in gaze deviation during visual distraction is due to the demands from the secondary task which requires drivers to sample information from some sort of visual display positioned in the vehicle, in addition to sampling information from the road scene. Changes to natural eye-movement patterns can lead to an increase in lateral deviation during such visual distraction. Godthelp, Milgram, and Blaauw (1984) argued that taking the eyes off the road causes an accumulation of heading errors, resulting in a more variable lateral position (as observed for example by steering reversals or standard deviation of lateral position). A different, but not dissimilar, explanation comes from the Active Gaze model of steering (Wilkie \& Wann, 2003; Wilkie, Wann, \& Allison, 2008), where gaze and steering are inexorably linked, with gaze direction being an input that directly feeds into the steering response.

In addition to changes in lateral position, some studies investigating the effect of visual distraction on driving have also reported changes in longitudinal control, such as speed reduction and longer headway to lead vehicles (e.g., Engström et al., 2005; Jamson \& Merat, 2005), but this is largely regarded as a compensatory strategy whereby drivers reduce the demands of the driving task by reducing their travel speed (Engström et al., 2005). There are also conflicting results in terms of the effect of visual distraction on drivers' response to discrete events, such as response to the brake lights of a lead vehicle, with Reyes and Lee (2008), for example, showing no effect on reaction time, whilst Hibberd, Jamson, and Carsten (2013) show a delay

\footnotetext{
${ }^{1}$ The term 'cognitive load' or 'cognitive task' is often used as a term to describe the latter (Lamble, Kauranen, Laakso, \& Summala, 1999; Engström, Johansson, \& Östlund, 2005; Jamson \& Merat, 2005). However, this creates an artificial dichotomy which implies that 'visual' tasks have no cognitive component(s); although there are visual tasks which can be void of cognitive components, these cannot be implemented in a driving scenario. Therefore the term 'non-visual' distraction will be used here to refer to tasks that do not have a visual component.
}

in brake reaction times with a concurrent visual distraction task.

Although the effects of visual distraction are relatively well-understood and documented, this is not the case with respect to the effects of nonvisually distracting tasks. While some studies have reported an increase in lateral deviation of the vehicle when using tasks without a visual component (e.g., Salvucci \& Beltowska, 2008; Strayer \& Johnston, 2001), what is most commonly observed is a reduction in the vehicle's lateral deviation (Atchley \& Chan, 2010; Cooper, MedeirosWard, \& Strayer, 2013; Engström et al., 2005; He, McCarley, \& Kramer, 2013; Jamson \& Merat, 2005; Kubose et al., 2006; Reimer, 2009), often accompanied by a reduction in the lateral deviation of gaze (Victor et al., 2005; Reimer, 2009). In terms of steering activity in particular, non-visual distraction has been reported to lead to more steering activity (such as increased steering wheel reversal rates and higher levels of high frequency steering) in some experiments (e.g. Engström et al., 2005; He et al., 2013; Kubose et al., 2006) but no change from baseline is observed in others (e.g., Jamson \& Merat, 2005). The relationship between measures that examine lateral deviation (e.g., Standard Deviation of Lane Position (SDLP)) and measures that quantify steering activity (such as steering wheel reversal rate (RR) and high-frequency steering (HFS)) is not straight-forward, however, and depends on a number of variables, including road geometry, and driver workload. For example, Medeiros-Ward, Cooper, and Strayer (2014) argue that because lane keeping is an automatic task (Michon, 1985) and does not necessarily require a focus of attention, it can actually benefit from diverted attention to a secondary task. However, even though lane-keeping may be considered an automatic task, and the reduction of the vehicle's lateral variability can be deemed an improvement in that task, engaging in secondary non-visual tasks is not necessarily beneficial to driving safety/performance. Detriments in detection of peripheral targets/events (e.g., Lee, Lee, \& Ng Boyle, 2007; Merat \& Jamson, 2008), harder braking events (Harbluk, Noy, Trbovich, \& Eizenman, 2007), and an increase in reaction times to critical events (Horrey \& Wickens, 2004) during non-visual secondary tasks have severe implica- 
tions to driver safety.

In terms of eye-movements, reductions in the lateral variability of gaze observed under conditions of non-visual distraction (Victor et al., 2005; Jamson \& Merat, 2005; Reimer, 2009), becomes more pronounced as the difficulty of the nonvisual task increases (Reimer, Mehler, Wang, \& Coughlin, 2010). Such distractions also result in drivers spending more time looking at the road ahead and directing less saccades towards the periphery (Harbluk et al., 2007; Recarte \& Nunes, 2000, 2003; Victor et al., 2005). This concentration of gaze towards the centre of the road under conditions of non-visual distraction is not yet fully understood, but one possible explanation which has been put forward is that drivers are prioritising the action task (i.e., driving/lane-keeping) which effectively treats gaze concentration as a compensation mechanism (Victor et al., 2005). Conversely, Recarte and Nunes (2000) argue that the gaze concentration could actually reflect the narrowing of the size of the attentional focus, which could also explain the detriments in detection of peripheral targets associated with non-visual distraction (Lee et al., 2007; Merat \& Jamson, 2008).

Recently, it has been argued that this concentration of gaze towards the road centre, by a nonvisual task, is amplified in car-following scenarios (Mühlbacher \& Krüger, 2011), since the lead car provides a point of focus for drivers. Mühlbacher and Krüger (2011) found that participants who followed a lead car showed lower lateral variability compared to participants who did not follow a lead car, although this study does not report any information about the pattern of eye-movements. One of the most influential models of how drivers use visual information to guide their steering is the two-point model of steering (Donges, 1978; Land \& Horwood, 1995; Salvucci \& Gray, 2004). As the name suggests, this model proposes that drivers use two salient points to drive: a far point that provides them with prospective (feed-forward) information about the road ahead, and a near point which provides them with feedback information about their position in the lane (Salvucci \& Gray, 2004). Whilst the near point can be sampled through peripheral vision, the far point requires gaze fixation. Based on the findings from Salvucci (2001) where drivers fixate on the car ahead (when one is present), Salvucci and Gray (2004) argue that the lead car acts as the far information point in that model.

In the present paper we examined the impact of visual and non-visual distraction on steering performance and gaze patterns in a number of driving conditions. The two secondary tasks used were a visual search task displayed on an in-vehicle information system (the Arrows task from the EU project HASTE, see Jamson and Merat (2005) for a description of the task) and a counting backwards in sevens task, which required no visual input. Whilst we expected steering and gaze variability to increase during the visual distraction task, we predicted that performance of the count backwards task would show greater gaze concentration towards the road centre and in turn lead to decreased steering variability.

In addition to the above, we included two further variables, in an attempt to understand the interaction between steering control, road geometry, eye movements, and secondary task performance. First, we wished to assess the effect of different driving environments on this interaction, suggesting that road geometry and curvature affect the demands placed on the human visuo-motor system. For example, more steering reversals are observed in curved roadways when compared to straight road sections, although the pattern of reversals has been found to be similar across visual and non-visual distraction tasks (Jamson \& Merat, 2005). It can be argued that maintaining perfect lateral control in curved road sections is more challenging, since the driver has to continuously adjust the position of the steering wheel to match the curvature of the road. However, when driving a straight road section, the steering task becomes a simple, more automated lanekeeping task, rather than curvature matching, per se. According to the two-point model of steering (Salvucci \& Gray, 2004) described above, simple lane keeping should rely more on feedback information while the curvature-matching task should rely on prospective as well as feedback information. By the same token, visually distracting tasks which take drivers' gaze away from the road will degrade steering control and lateral position accuracy, whether the path to be followed is straight or curved. However, as road curvature increases, the 
interaction between the nature of the secondary task and steering control becomes more complicated.

Finally, to further understand the interaction between distracting tasks, steering control, and gaze direction, we also manipulated the presence (Experiment 1) and behaviour (Experiment 2) of a lead car on the road. We argued that during the counting backwards task the presence of a lead car should enable more concentration of gaze in the centre of the road (and on the lead vehicle) compared to the conditions without a lead car. This, in conjunction with the two-point model of steering (Salvucci \& Gray, 2004) which argues that the lead car acts as the far point for prospective control, would indicate that under conditions of non-visual distraction, drivers would be more influenced by the steering behaviour of the lead vehicle. To test this, in Experiment 2 we had conditions where a lead car would follow a 'perfect path' in the centre of the lane and compared performance with conditions where the lead car followed a sinusoidal path within the lane. The interaction between these factors and road geometry was also examined.

\section{Methods}

\section{Participants}

All testing adhered to the ethical guidelines laid out by the University of Leeds Research Ethics Committee. A within-subjects design was used for both experiments. Fifteen participants were recruited for each experiment using the University of Leeds Driving Simulator (UoLDS) database, and all participants held a valid UK driving licence for a minimum of 4 years. The average age of participants in Experiment 1 was $29.6 \pm 10.73$ years, and out of the 15 participants, eight of them were males. The average age of participants in Experiment 2 was $33.4 \pm 8.03$ years, and out of the 15 participants, eight of them were females. Participants were reimbursed for their time with $£ 15$ in cash.

\section{Design and Procedure}

\section{Materials}

Both experiments were conducted in the UoLDS which consists of a Jaguar S-type cab with all driver controls operational. The vehicle is housed within a $4 \mathrm{~m}$ spherical projection dome and has a $300^{\circ}$ field-of-view projection system. A v4.5 Seeing Machines faceLAB eye-tracker was used to record eyemovements at $60 \mathrm{~Hz}$.

\section{Driving Environment}

For both experiments, participants were given a 20-minute familiarisation drive, and each experiment consisted of four experimental drives. Each of the drives consisted of a rural, two lane road with Straight road and Curved sections, each of which were approximately $7.5 \mathrm{~km}$ long. The width of each lane was $3.65 \mathrm{~m}$. The Straight and Curved rural sections were separated by a short urban environment where no data were collected. The curved sections consisted of 30 bends (15 left, 15 right) and each bend had a radius of $750 \mathrm{~m}$. The speed of the lead car (when present) was $22.3 \mathrm{~m} / \mathrm{s}$ $(80.5 \mathrm{~km} / \mathrm{h} ; 50 \mathrm{mph})$, in both experiments. In Experiment 2 , the lead car either followed a smooth or sinusoidal path which oscillated around the centre of the lane with a maximum offset of $0.8 \mathrm{~m}$. Each experimental drive lasted approximately 20 minutes.

\section{Distraction Tasks}

Two distraction tasks were used in these experiments, a counting-backwards task (non-visual distraction/Numbers task) and a visual search task (visual distraction/Arrows task). For the Numbers task participants heard a series of 3-digit numbers through the car's speakers and were asked to count backwards in steps of seven until they heard a "beep" tone indicating they should stop. The interval from the presentation of the 3-digit number to the "beep" tone was 30 seconds.

The visual-search task (Arrows) used a subset of the Arrows task used in the HASTE project (see Jamson \& Merat, 2005). Participants were shown a $4 \times 4$ grid with arrows of mixed orientation on an in-vehicle touchscreen display mounted on their 
left just beneath the windshield. Participants had to indicate whether a target arrow (always an arrow pointing upwards) was present in the display or not by clicking the YES or NO button on the touch-screen. Half of the displays contained the target arrow and once participants entered their response a new grid was shown. Each grid presentation was accompanied by a short auditory signal and the total length of each of the Arrows task trials was also 30 seconds.

In addition to collecting data during the two distracting tasks, data were collected from baseline conditions where participants did not engage in a secondary task.

\section{Design}

Three factors were considered for each experiment (Lead car, Road, and Task). In Experiment 1 there were two levels of Lead car (Lead car, No Lead car), two levels of road (Straight, Curve) and three levels of Task (Baseline, Arrows, Numbers), therefore a total of 12 conditions were included, with each condition repeated twice. Conditions for Experiment 1 are shown in Table 1.

Each of the four drives consisted of a Straight road section followed by a Curved road section (Curve). Each drive included eight Task trials (four in the Straight section and four in the Curved section). The Tasks started once the drivers exited the initial urban environment and reached the rural two-lane road. In Experiment 2 there were two levels of Lead car (Lead car, Sinusoidal Lead car), two levels of road (Straight, Curved) and three Task levels (Baseline, Arrows, Numbers).The conditions and drives were ordered in the same way as in Experiment 1 .

\section{Measures}

In terms of driving metrics, we report measures of Standard Deviation of Lateral position (SDLP), steering wheel reversal rates equal or greater to three degrees (SRRs), Mean Speed, and Mean Headway. SDLP measures the variation of lane position, typically indicating a measurement of how accurately drivers manage to maintain their target lane position. SDLP essentially provides an index for road tracking error and ability to control the lateral motion of the vehicle (e.g., Allen
\& O’Hanlon, 1979). Steering Reversal Rates are a measure of corrective steering and measured as changes in steering wheel angle that are equal to or greater than 3 degrees.

In terms of eye-movements, we looked at the Standard Deviation of Yaw angle (SD Yaw), which measures drivers' lateral scanning pattern of the scene (high values) or concentration towards the road ahead (low values). Although gaze variability can also be examined by combining the lateral (yaw) and vertical (pitch) variability scores (see Victor et al., 2005), here we focused on lateral deviation since it is a better measure of gaze concentration towards the centre of the road and overall spread of gaze around the scene (Reimer, 2009; Wang et al., 2014). We also looked at the mean pitch angle (Mean Pitch).

\section{Results and Discussion}

\section{Experiment 1: Lead Car vs No Lead}

In this experiment, we examined the effect of visual and non-visual distraction on lateral control measures during straight and curved road sections, with or without a lead vehicle. Since non-visual distraction is shown to reduce gaze concentration, we hypothesised that if the lead vehicle is used as a focus point for gaze, then one would expect higher gaze concentration towards the road centre around the location of the lead vehicle (i.e., lower SD Yaw) when drivers were asked to count backwards and follow the lead car. In the absence of the lead car, counting backwards would still reduce SD Yaw compared to baseline, but not compared to when a point of focus was present in the form of a lead car (as argued by Mühlbacher and Krüger (2011). By the same token, based on the relationship between gaze concentration and steering control, we expected lower values for SDLP when counting backwards was conducted with the lead car present. The interaction between these measures and road geometry was also examined.

\section{Eye-movements}

Out of the 15 participants tested for this experiment, eight provided adequate eye-movement 
Table 1 Conditions for Experiment 1. Each Task lasted for 30 seconds; all conditions where counterbalanced. Experiment 2 was identical, but the 'No Lead' conditions were substituted by 'Sinusoidal Lead'.

\begin{tabular}{rllllll} 
& \multicolumn{3}{c}{ STRAIGHT } & & CURVE & \\
LEAD & Baseline & Arrows & Number & Baseline & Arrows & Number \\
NO LEAD & Baseline & Arrows & Number & Baseline & Arrows & Number
\end{tabular}

data, as assessed by the FaceLab eye-tracker software, which provides a confidence level of $0-3$. Therefore, for the purpose of gaze analyses, $N=8$.

\section{Eye-movements: Mean Pitch}

A 3 (Task: Baseline, Arrows, Numbers) $\times 2$ (Lead, No Lead) $\times 2$ (Road: Straight, Curves) repeatedmeasures ANOVA was carried out on the Mean Pitch of gaze angle. This yielded a significant main effect of Task $\left(F(2,14)=59.36, p<.001, \eta_{p}^{2}=\right.$ $.89)$, as well as a significant main effect of Road $\left(F(1,7)=18.93, p=.003, \eta_{p}^{2}=.73\right)$; no other significant main effects or interactions were found for this measure.

The main effect of task was analysed us ing pairwise-comparisons with LSD adjustment where all comparisons yielded significant results, Baseline $(\bar{x}=0.843$, SEM $=0.48)$ vs Arrows $(\bar{x}=$ -4.456 , SEM $=0.44) p<.001$, Arrows vs Numbers $(\bar{x}=2.218$, SEM $=0.49) p<.001$, and Baseline vs Numbers $p=.005$.

On average, participants looked lower down during the Arrows condition, towards the in-vehicle display and during the Numbers condition participants' vertical gaze angle was higher when compared to Baseline.

The main effect of Road is caused by a significant difference in mean pitch between straight $(\bar{x}=-.929, \mathrm{SEM}=.302)$ and curved $(\bar{x}=-.001$, $\mathrm{SEM}=.314$ ) sections of the road. Overall, participants looked lower down on the straight sections of the road, compared to the curved sections. As the driving task became more demanding in the curved sections, participants' gaze was directed higher up in the horizon, looking further ahead towards their future path. This finding supports other studies which have shown that looking further ahead provides drivers with prospective information about the road and its curvature (Land \& Horwood, 1995; Salvucci \& Gray, 2004).

\section{Eye-movements: SD Yaw}

A 3 (Task: Baseline, Arrows, Numbers) $\times 2$ (Lead, No Lead) $\times 2$ (Road: Straight, Curves) repeatedmeasures ANOVA on the Standard Deviation of gaze yaw angle showed a significant main effect of Task $\left(F(2,14)=102.31, p<.001, \eta_{p}^{2}=.94\right)$, a significant interaction between Task and Road $\left(F(2,14)=6.94, p=.008, \eta_{p}^{2}=.50\right)$, as well as a significant interaction between all three factors $\left(F(2,14)=3.85, p=.046, \eta_{p}^{2}=.35\right)$, as shown in Figure 1.

Simple effects analysis on the triple interaction between Task, Road and Lead shows that when drivers were following the Lead car (left panel in Figure 1) there were no significant differences in SD of gaze yaw angle between the two road conditions across the three levels of Task (Baseline: $p=.814$, Arrows: $p=.179$, Numbers: $p=.082$ ). However in the No Lead conditions (right panel in Figure 1) there was a difference in gaze concentration between the road conditions during the Numbers task ( $p=.004)$ - with lower SD Yaw in the Straight road conditions. SD Yaw was not found to be significantly different for Straight and Curved road sections during both the Arrows ( $p=.141$ ) and Baseline conditions ( $p=.064$ ). It appears, therefore, that an increase in gaze concentration with concurrent performance on the non-visual Numbers task existed whether or not a Lead car was present, a finding in contrast with the predictions of Mühlbacher and Krüger (2011). Therefore, drivers did not necessarily use the Lead vehicle as a focal point during these conditions, and engagement in the demanding non-visual task simply increased gaze concentration towards a focal area somewhere on the road ahead of their own vehicle.

\section{Vehicle Measures: SDLP}

The 3 (Task: Baseline, Arrows, Numbers) $\times 2$ (Lead, No Lead) $\times 2$ (Road: Straight, Curves) repeated- 

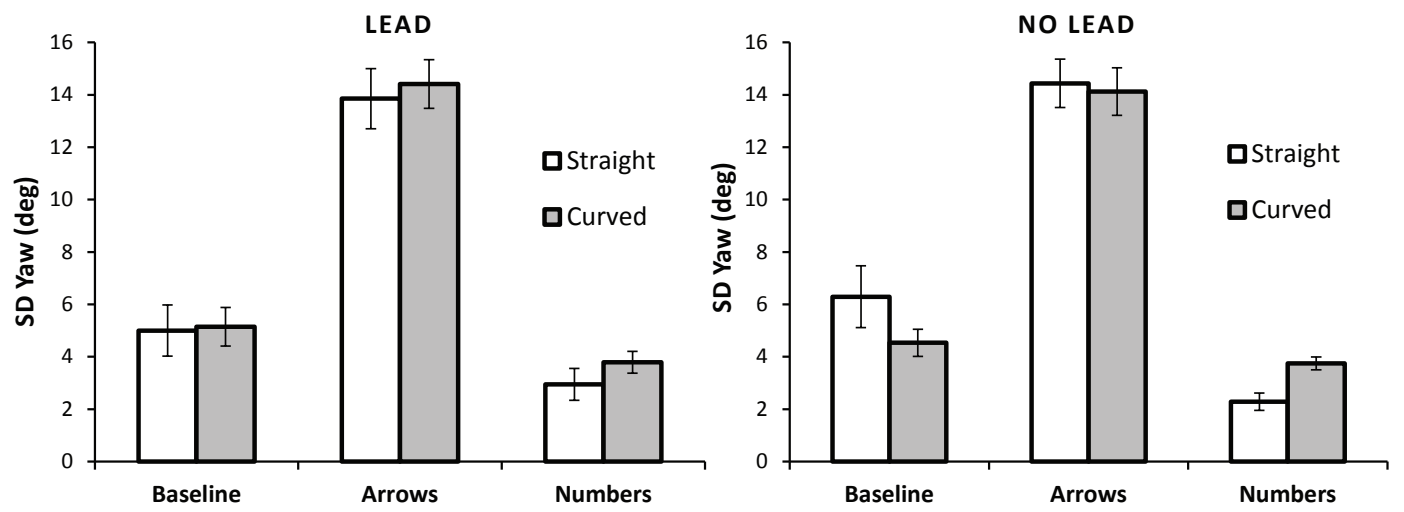

Figure 1 The triple interaction between Task, Road and Lead car for the Standard Deviation of gaze yaw angle. Error bars $=$ SEM.

measures ANOVA on standard deviation of lateral position showed a main effect of Task, Road and Lead car presence but did not reveal any significant interactions between these factors.

The main effect of Task $(F(2,28)=59.89, p<$ $.001, \eta_{p}^{2}=.81$ ) is shown in Figure 2. This effect was analysed with pairwise-comparisons with LSD adjustment with all comparisons showing significant differences between the three Task conditions at the $p<.001$ level. In agreement with previous studies, results showed the highest levels of SDLP during the Arrows condition and the lowest SDLP in the Numbers condition. This finding is partly in line with the gaze data, which showed reduced gaze variability during the Numbers task and can be explained by the Active Gaze model of steering (Wilkie \& Wann, 2003; Wilkie et al., 2008) where gaze and steering are interdependent.

SDLP was found to be lower in the presence of the Lead $\operatorname{car}\left(F(1,14)=16.86, p=.001, \eta_{p}^{2}=.55\right)$ $(\bar{x}=.187, \mathrm{SEM}=.010)$ compared to the No Lead car conditions $(\bar{x}=.210$, SEM $=.009)$. This finding was observed irrespective of road geometry or concurrent task type. It can be argued that these results are in line with the two-point model of steering where the Lead car is used as the prospective information point and has a stabilising effect on steering control.

The main effect of Road $(F(1,14)=60.42, p<$ $\left..001, \eta_{p}^{2}=.81\right)$ is clearly caused by lower levels of SDLP during the Straight road conditions $(\bar{x}=.140$, $\mathrm{SEM}=.006)$ compared to the Curved road sections

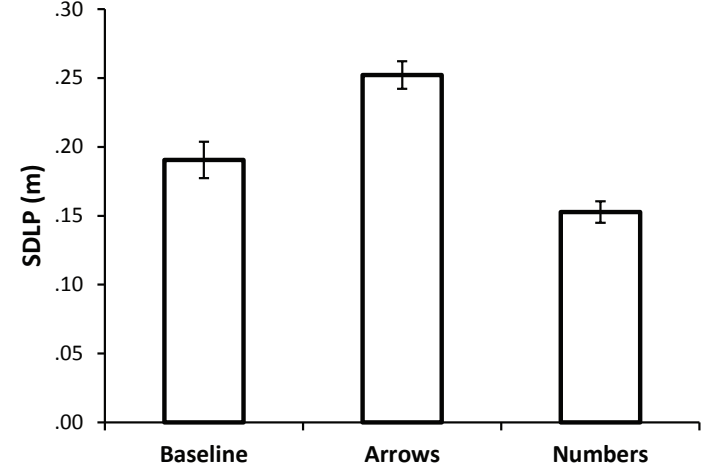

Figure 2 The main effect of Task condition in standard deviation of lateral position. Error bars = SEM.

$(\bar{x}=.257, \mathrm{SEM}=.016)$.

\section{Vehicle Measures: SRRs}

A 3 (Task: Baseline, Arrows, Numbers) $\times 2$ (Lead, No Lead) $\times 2$ (Road: Straight, Curves) repeatedmeasures ANOVA was run for the 3 degree reversal rates, which showed significant main effect for Task $\left(F(2,28)=16.69, p<.001, \eta_{p}^{2}=.54\right)$, Lead $\left(F(1,14)=16.72, p=.001, \eta_{p}^{2}=.54\right)$ and Road $\left(F(1,14)=144.88, p<.001, \eta_{p}^{2}=.91\right)$. There was a significant interaction between Lead and Road $\left(F(1,14)=10.42, p=.006, \eta_{p}^{2}=.43\right)$; no other interactions reached significance.

The main effect of Task (shown in Figure 3) was 
analysed with LSD comparisons; Arrows produced significantly higher reversal rates compared to Baseline $(p<.001)$ and Numbers $(p=.010)$. Rever sals for Numbers were not significantly different to Baseline, although the comparison did approach significance $(p=.056)$.

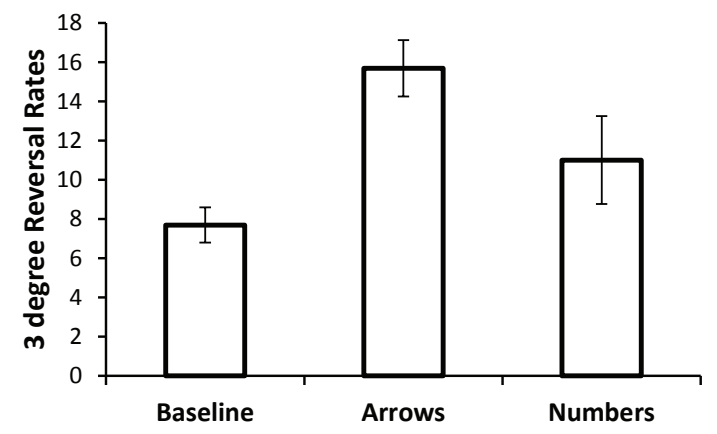

Figure 3 Steering wheel Reversal Rates for the main effects of Task. Error bars = SEM.

The interaction between Road and Lead is shown in Figure 4, and is driven by higher SRRs in the Curved roads when there is no Lead car. This indicates that the presence of the Lead car can have a stabilisation effect on steering control, similar to that reported for SDLP.

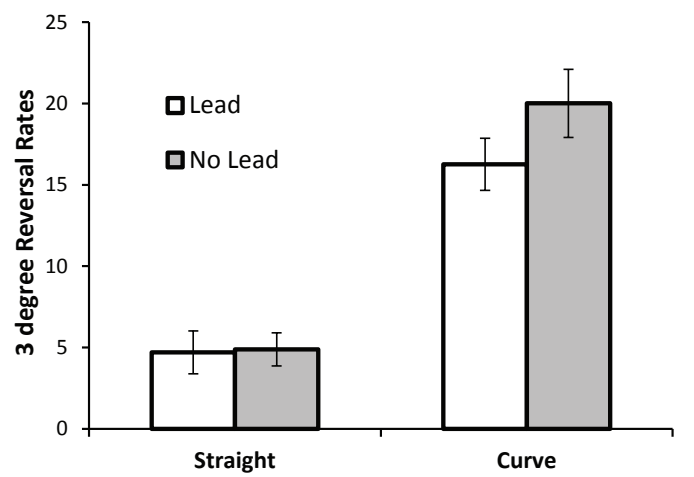

Figure 4 The interaction between Lead car and Road in 3 degree reversal rates. Error bars $=$ SEM.

\section{Vehicle Measures: Mean Speed}

A 3 (Task: Baseline, Arrows, Numbers) $\times 2$ (Lead, No Lead) $\times 2$ (Road: Straight, Curves) repeatedmeasures ANOVA was run for the average speed, and showed significant main effects for Task, Lead, and Road, Task $\times$ Road, Task $\times$ Lead, Lead $\times$ Road, as well as a significant interaction between all three factors $\left(F(2,28)=15.56, p<.001, \eta_{p}^{2}=.53\right)$, shown in Figure 5.

Simple main effects analysis on the triple interaction between Task, Lead, and Road revealed that there were no significant differences in speed between the three Task conditions when drivers were behind the Lead car on the Straight roads. However, in the Curved road sections, speed was significantly lower during the Arrows task than both Baseline $(p=.002)$ and Numbers $(p=.002)$; no significant difference was found between Baseline and Numbers for the Lead conditions ( $p=.959)$.

In the No Lead conditions, on Straight roads, participants sped up significantly during the Numbers task compared to both Baseline $(p<.001)$ and Arrows task ( $p=.001$ ) while no difference was found between Baseline and Arrows ( $p=.709$ ). However, on the Curved road sections, during both the Arrows and Numbers tasks, participants slowed down compared to Baseline (Arrows vs Baseline: $p<.001$; Numbers vs Baseline: $p=.020$ ) while no significant difference in speed was found between Arrows and Numbers tasks ( $p=.122$ ).

Not surprisingly, the presence of a Lead car limited participants' speed. However, there was a distinction in speed between Straight and Curved roadways, with participants slowing down on the Curved roadways when performing the Arrows task. This might be considered a compensation mechanism, where drivers were perhaps aware of their limitations in performing the two tasks together, when they were required to look away from the road during the Arrows task. However, this limitation was clearly not perceived by drivers during performance of the Numbers task.

In the No Lead conditions, the distinction between road geometry becomes even more apparent: when driving around bends, drivers reduced their speed on both the Arrows and Numbers tasks, compared to Baseline. However, when speed was not restricted by a Lead car, participants drove significantly faster when performing the Numbers task on the less challenging Straight road sections. Taken together, the data shows a clear interaction between road geometry, speed of travel and the nature of the secondary tasks, such that when a 

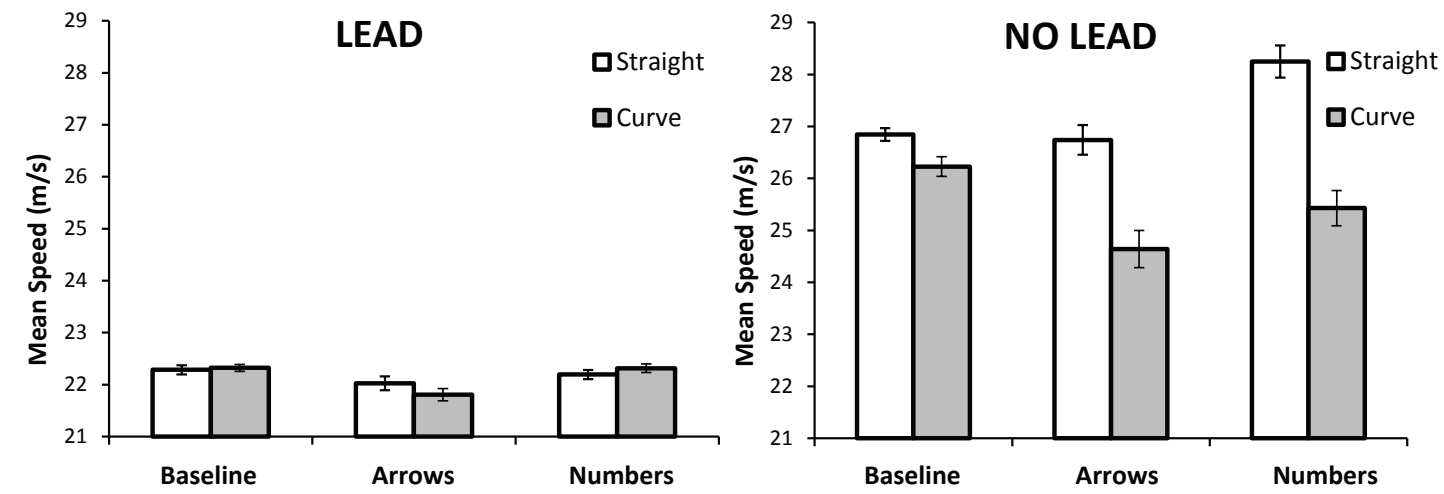

Figure 5 The significant interaction between Task, Road, and Lead in Mean Speed. Error bars = SEM.

Lead car is restricting their speed, drivers are only aware of their limitations in secondary task performance during the more difficult Curve sections, but consider the Straight sections easier to manage and do not appreciate the consequences of conducting the nonvisual Numbers task on their speed and subsequent safety.

\section{Experiment 2: Normal Lead vs Sinu- soidal Lead}

Experiment 1 showed that the presence of a Lead car had a stabilising effect on steering, as measured both by SDLP and 3 degree SRRs. However, gaze concentration towards the centre of the road was more pronounced during the Numbers task, regardless of Lead car presence.

In Experiment 2, the same Task and Road conditions were used as in Experiment 1, but we manipulated the path of the Lead car to better understand the relationship between Lead car presence, eye-movements and steering control, with and without secondary task performance. Here, we manipulated the path followed by the Lead car, where performance following a Lead car with a 'perfect path' in the centre of the lane (Normal Lead) was compared to that following a sinusoidal path (Sinusoidal Lead). We predicted that if drivers use the Lead car as a far information point, the stabilisation effect of the Lead car observed when performing the Numbers task in Experiment 1 would not be replicated, and instead an increase in SDLP should be observed when drivers were following the Sinusoidal Lead.

\section{Eye-movements}

Out of the 15 participants tested for this experiment, ten provided adequate eye-movement data (maximum quality for more than half of the frames of interest), as assessed by the FaceLab eye-tracker software, which provides a confidence level of 0 3 . Therefore, for the purpose of gaze analyses, $N=10$.

\section{Eye-movements: Mean Pitch}

A 3 (Task: Baseline, Arrows, Numbers) $\times 2$ (Lead: Normal, Sinusoidal) $\times 2$ (Road: Straight, Curves) repeated-measures ANOVA was carried out on the mean pitch of gaze angle. As in Experiment 1, there was a significant main effect of Task $(F(2,18)=$ 46.27, $\left.p<.001, \eta_{p}^{2}=.84\right)$, as well as a significant main effect of $\operatorname{Road}\left(F(1,9)=60.35, p<.001, \eta_{p}^{2}=\right.$ $.87)$. No other significant effects or interactions were found.

The main effect of Task was analysed using pairwise-comparisons with LSD adjustment, and all comparisons yielded significant results (Baseline $(\bar{x}=-2.392$, SEM $=0.76)$ vs Arrows $(\bar{x}=$ -6.869 , SEM $=0.88), p<.001$; Arrows vs Numbers $(\bar{x}=-1.559$, SEM $=0.68), p<.001$; Baseline vs Numbers, $p=.022$ ), as in Experiment 1.

The pattern of this mean pitch angle of gaze was the same in both experiments, with participants looking lower - towards the in-vehicle display - 

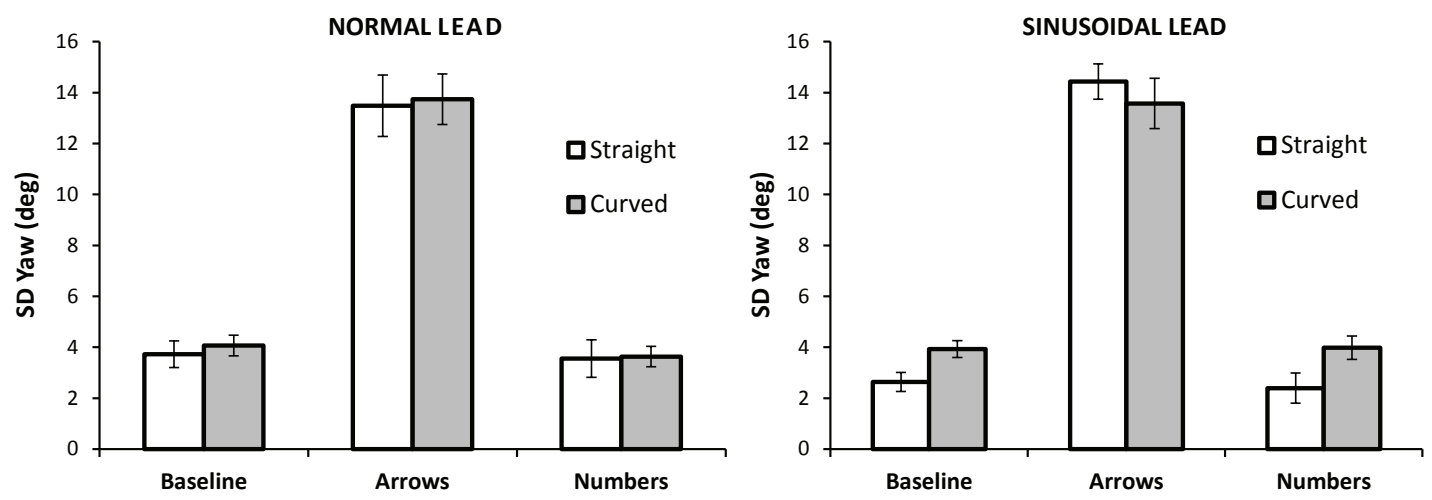

Figure 6 The triple interaction between Task, Road and Lead car in the Standard Deviation of gaze yaw angle. Error bars $=$ SEM.

during the Arrows condition and higher during the Numbers when compared to Baseline.

The main effect of Road is again caused by drivers looking lower during the Straight roads $(\bar{x}=-4.151, \mathrm{SEM}=.750)$ compared to the Curved roads $(\bar{x}=-3.062$, SEM $=.652)$.

\section{Eye-movements: SD Yaw}

A 3 (Task: Baseline, Arrows, Numbers) $\times 2$ (Lead: Normal, Sinusoidal) $\times 2$ (Road: Straight, Curves) repeated-measures ANOVA was carried out on the Standard Deviation of gaze yaw angle. There was a significant effect of Task $(F(2,18)=91.48, p<$ $\left..001, \eta_{p}^{2}=.91\right)$, a significant interaction between Task and $\operatorname{Road}\left(F(2,18)=4.28, p=.030, \eta_{p}^{2}=.32\right)$, as well as a significant interaction between all three factors $\left(F(2,18)=5.54, p=.013, \eta_{p}^{2}=.38\right)$.

The triple interaction between Task, Road and Lead was analysed using simple main effects. In the Normal Lead car conditions (Figure 6, left panel) there were no significant differences between the Road conditions at any level of Task (Baseline: $p=.525$; Arrows: $p=.530$; Numbers: $p=.891$ ). In the Sinusoidal Lead conditions (Figure 6 , right panel), although there was no difference in SD Yaw between the two Road conditions when drivers performed the Arrows task ( $p=.237$ ), SD Yaw was lower in the Straight Road sections both during Baseline driving $(p<.001)$ and when participants completed the Numbers task $(p=.002)$.
Standard deviation of yaw during the Normal Lead conditions was therefore similar to Experiment 1 (Figure 1). However, when participants were required to follow a Lead car with a sinusoidal trajectory, a higher concentration of gaze was observed in the straight road sections during both the Baseline and Numbers conditions. As in Experiment 1 , there was no effect of the distracting tasks on SD Yaw during the curved road sections. Therefore, sinusoidal movement of the Lead car did not seem to alter the pattern of eye movements either during single task driving or with the addition of a secondary task, when results were compared to that of the Lead car following a perfect travel path ahead of drivers.

\section{Vehicle Measures: SDLP}

A 3 (Task: Baseline, Arrows, Numbers) $\times 2$ (Lead: Normal, Sinusoidal) $\times 2$ (Road: Straight, Curves) repeated-measures ANOVA was carried out on the SDLP. Similar to Experiment 1, there was a significant main effect of Task $(F(1.387,19.416)=$ $\left.34.38, p<.001, \eta_{p}^{2}=.71\right)$ and a significant main effect of $\operatorname{Road}\left(F(1,14)=77.74, p<.001, \eta_{p}^{2}=.84\right)$, but no significant effect of Lead car and no significant interactions between any of the factors.

The main effect of Task shown in Figure 7 was analysed using pairwise-comparisons, with LSD adjustment, and all comparisons yielded significant results (Baseline vs Arrows, $p<.001$; Arrows vs Numbers, $p<.001$; Baseline vs Numbers, 
$p=.011)$. Therefore, as in Experiment 1, the Arrows tasks produced the highest deviation in lane and the Numbers tasks the lowest, regardless of the behaviour of the Lead car.

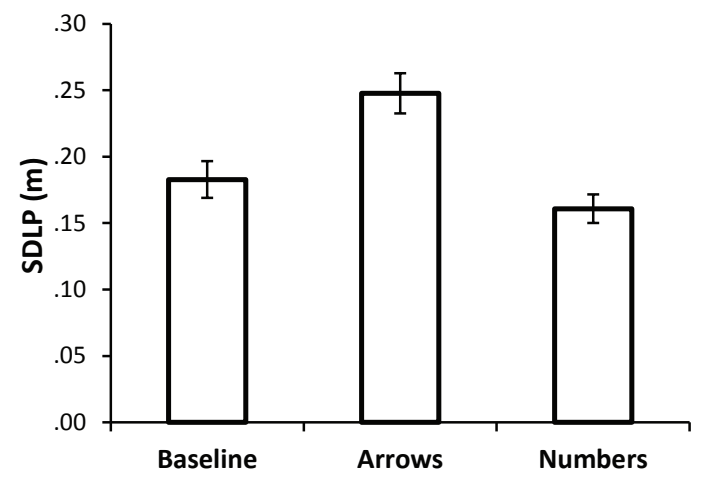

Figure 7 The main effect of Task in SDLP. Error bars = SEM.

The main effect of Road was caused by higher lane deviation on Curved roads $(\bar{x}=.245$, SEM $=$ .014 ) compared to Straight roads $(\bar{x}=.149$, SEM $=$ .012), in line with Experiment 1.

\section{Vehicle Measures: SRRs}

A 3 (Task: Baseline, Arrows, Numbers) $\times 2$ (Lead: Normal, Sinusoidal) $\times 2$ (Road: Straight, Curves) repeated-measures ANOVA was carried out on 3 degree SRRs. There was a main effect of Task $\left(F(2,28)=17.63, p<.001, \eta_{p}^{2}=.56\right)$, and a main effect of $\operatorname{Road}\left(F(1,14)=299.47, p<.001, \eta_{p}^{2}=.95\right)$. No other main effect or interaction reached significant levels.

The main effect of Task (shown in Figure 8) follows the same pattern as Experiment 1, with Arrows producing significantly higher reversal rates compared to both Baseline $(p<.001)$ and Numbers $(p=.002)$, while no significant difference was found between Baseline and Numbers ( $p=.143$ ).

The main effect of Road was caused by significantly lower SRRs on Straight roads $(\bar{x}=4.29$, SEM $=1.08$ ) compared to Curved roads ( $\bar{x}=13.39$, SEM $=1.10$ ). This effect is explained by the road geometry characteristics.

The main effect of Task is the same as that found in Experiment 1. Interestingly, although the presence/absence of a Lead car in Experiment 1 did

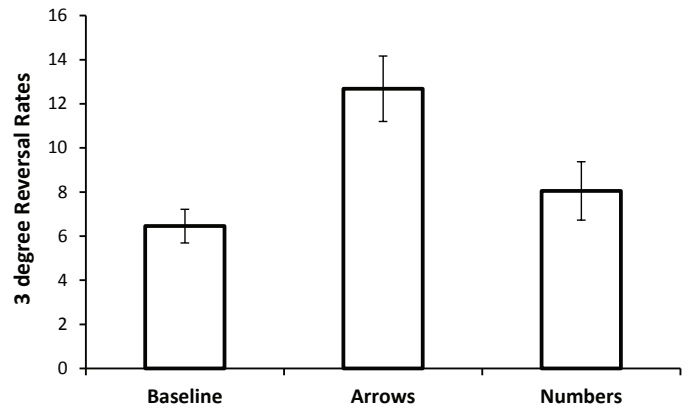

Figure 8 The main effect of Task on 3 degree reversal rates. Error bars $=$ SEM.

affect SRRs (with the Lead car providing a stabilising effect on steering), the sinusoidal trajectory of the Lead car used in this experiment had no effect on SRRs, which suggests that that drivers were perhaps ignoring unreliable sources of information from the lead vehicle.

\section{Vehicle Measures: Mean Speed}

A 3 (Task: Baseline, Arrows, Numbers) $\times 2$ (Lead: Normal, Sinusoidal) $\times 2$ (Road: Straight, Curves) repeated-measures ANOVA was carried out on mean speed, and results showed a significant main effect of Task $\left(F(2,28)=20.02, p<.001, \eta_{p}^{2}=\right.$ $.59)$, but no other main effects or interactions approached significance.

The main effect of Task was analysed using LSD comparisons. During the Arrows task $(\bar{x}=21.66$, SEM = .11), participants slowed down significantly compared to Baseline $(\bar{x}=22.41$, SEM $=.09)$, $p=.001)$, and Numbers $(\bar{x}=22.55, \mathrm{SEM}=.12)$, $p<.001$. No difference was found between Numbers and Baseline, $p=.206$. The pattern of only slowing down during the Arrows task found here is the same as when the Lead car was present in Experiment 1 . Therefore, the Sinusoidal Lead conditions appeared to have no impact on participants' speed profile.

\section{General Discussion}

Our main aim in these experiments was to further understand the interaction between the effect of visual and non-visual distraction tasks on 
lateral control in driving, and examine whether road geometry, Lead car presence and behaviour of the Lead car affect eye-movement behaviour and hence lateral and longitudinal vehicle control. The same two distraction tasks were used in both experiments (a visual search task and a counting backwards task) and driving performance and eye movements were compared to when driving was done with no secondary task (Baseline). While the first experiment examined steering in the presence or absence of a Lead car, the second experiment used a normal Lead car (which followed a trajectory keeping a central lane position) and compared performance to following a lead car which obeyed a lateral sinusoidal path within the lane.

Results suggest that the two distraction tasks have different effects on gaze patterns and steering control, as shown by both experiments and in line with previous experiments of this nature (Engström et al., 2005; Jamson \& Merat, 2005; Merat \& Jamson, 2008). Quite predictably, since drivers looked towards the display screen during the Arrows task, results showed higher standard deviation of yaw gaze angle, accompanied by higher standard deviation of lateral position (a measure of steering performance). This finding is in line with the Active Gaze model of steering, where eyemovements are inexorably linked to steering patterns (Wilkie \& Wann, 2003; Wilkie et al., 2008). Gaze patterns in the Numbers task, which did not require any visual input, showed more concentration (lower SD yaw angle) compared to baseline. This was mirrored again in steering patterns, with a decrease in lane position variability. Although this difference in steering patterns may be as a result of the secondary task used, the above findings could also be attributed to differences in gaze patterns. We argue that in order to examine the pure effect of a distraction task on steering, gaze direction should be taken into account (Kountouriotis, Wilkie, Gardner, \& Merat, 2015), and show in a recent study that this increase in steering variability by a visual task is abolished if the task is placed on the driving scene itself (Merat, Kountouriotis, Tomlinson, \& Carsten, 2015). On the other hand, recent studies suggest that the reduced lateral deviation and improved lane keeping observed in the presence of a concurrent cognitive task may be due to the engagement of attentional resources by the cognitive task, which then prevents a topdown interference with highly automatised tasks such as lane keeping (Cooper et al., 2013). While a decrease in lane variability can be considered better driving performance, this is not necessarily the case, particularly if it is coupled with a decrease in lateral eye-movements. Such behaviour can be characterised as more rigid steering, and coupled with the reduction in lateral eye-movements could indicate that drivers will be worse at hazard perception of objects in the peripheral view and the ability to avoid collisions in that space. However, since reaction to hazards in the periphery was not directly tested in the experiments reported here, such a prediction should be treated with caution.

In terms of the effect of the lead car manipulation on eye-movements and steering control, based on the two-point model of steering (Salvucci \& Gray, 2004), we expected that in Experiment 1 gaze would focus on the lead car, if present, and be more diverse in the No Lead car condition. Any such increase in gaze concentration would be highest when participants were engaged in the nonvisual distraction task (as proposed by Mühlbacher \& Krüger, 2011). We also predicted that in Experiment 2 , steering variability would be reflected by the sinusoidal path of the lead car in the sinusoidal lead conditions. Experiment 1 showed that when drivers were following a lead car, there were no significant differences in gaze variability between road conditions at any level of Task (which was not the case in the No Lead conditions), indicating that drivers could indeed be using the lead car as an information point. However, the second prediction did not hold, since in Experiment 2 we did not observe an interaction between Task and Lead Car in either steering or eye-movement patterns. We expected that, if drivers did use the lead car as the 'far point' to gain feed-forward information (Salvucci \& Gray, 2004; Salvucci, 2001), their steering variability would reflect that pattern and increase in the sinusoidal lead conditions, and even more so under conditions of driver distraction. However, the path followed by the lead car in Experiment 2 had no significant effect on either steering reversals or steering variability. Drivers were therefore able to overlook the sinusoidal pattern of the lead vehicle. It remains to be seen how performance is affected by either a more erratic si- 
nusoidal deviation of the lead vehicle, or by a more subtle manipulation of the lead car's trajectory.

Macdonald and Hoffmann (1980) argue that steering reversals drop when a concurrent task is added, because participants remove their attention from the steering task. Here, we found no change in three degree reversal rates during the Numbers task, compared to Baseline, in either experiment. However, when drivers had to take their eyes off the road to complete the Arrows task, this was accompanied by an increase in three-degree reversal rates, which is likely to be due to corrections for heading errors. Hoffman and colleagues suggest there to be a complicated relationship between steering reversals and distraction task and suggest reversal rates "represent control effort, rather than an absolute measure of tracking performance" (p. 735).

The driving scene comprised of both straight and curved sections, which were analysed for performance separately, rather than collapsed across. This approach led to some interesting findings in terms of drivers' performance on secondary tasks in different driving environments and the interactions between eye-movements, steering behaviour and speed control. For example, in the absence of a Lead Car in Experiment 1, when drivers' speed was not restricted, participants drove at a higher speed during the easier Straight road sections, but reduced their speed in the more challenging Curved sections. However, the interaction of speed and secondary tasks suggest that although participants seem to have appreciated the negative effects of conducting the Arrows task during Curved sections and reduced their speed in order to compensate for such distractions, they were perhaps not as concerned about the effect of the Numbers task on driving performance and maintained a higher speed even in the Curve sections. This indicates that in more demanding environments driving is prioritised over secondary tasks, especially if drivers are aware of their limitations in dual tasking, for example when their eyes are taken away from the road. These results may also explain some inconsistencies found in the literature on the effects of distractions on driving, however should be treated with caution due to the relatively small effects.

In conclusion, the effect of driver distraction on eye-movements, speed control and steering performance can be influenced by environmental factors such as road curvature and also by the presence of other vehicles. Therefore the consequence of such interactions should be considered when assessing the effect of in-vehicle tasks on road safety.

Acknowledgments: GK was funded by EPSRC EP/J002933/1 (FORWARN) as a Research Fellow. The EPSRC had no involvement in the design, data collection, analysis and interpretation, or the writing of this manuscript.

\section{References}

Allen, R. W., \& O’Hanlon, J. F. (1979). Effects of roadway delineation and visibility conditions on driver steering performance. Transportation Research Record, 739, 5-8.

Atchley, P., \& Chan, M. (2010). Potential Benefits and Costs of Concurrent Task Engagement to Maintain Vigilance: A Driving Simulator Investigation. Human Factors: The Journal of the Human Factors and Ergonomics Society, 53(1), 3-12.

Baddeley, A. (1992). Working memory. Science, 255(5044), 556-559.

Cooper, J. M., Medeiros-Ward, N., \& Strayer, D. L. (2013). The Impact of Eye Movements and Cognitive Workload on Lateral Position Variability in Driving. Human Factors: The Journal of the Human Factors and Ergonomics Society, 55(5), 1001-1014.

Donges, E. (1978). A two level model of driver steering behavior. Human Factors, 20(6), 691-707.

Engström, J., Johansson, E., \& Östlund, J. (2005). Effects of visual and cognitive load in real and simulated motorway driving. Transportation Research Part F: Traffic Psychology and Behaviour, 8(2), 97-120.

Godthelp, H., Milgram, P., \& Blaauw, G. J. (1984). The development of a time-related measure to describe driving strategy. Human Factors, 26(3), 257-268. 
Groeger, J. A. (2000). Understanding Driving: Applying Cognitive Psychology to a Complex Everyday Task. Psychology Press.

Harbluk, J. L., Noy, Y. I., Trbovich, P. L., \& Eizenman, M. (2007). An on-road assessment of cognitive distraction: impacts on drivers' visual behavior and braking performance. Accident; analysis and prevention, 39(2), 3729.

He, J., McCarley, J. S., \& Kramer, A. F. (2013). Lane Keeping Under Cognitive Load: Performance Changes and Mechanisms. Human Factors: The Journal of the Human Factors and Ergonomics Society, 56(2), 414-426.

Hibberd, D. L., Jamson, S. L., \& Carsten, O. M. J. (2013). Mitigating the effects of in-vehicle distractions through use of the Psychological Refractory Period paradigm. Accident; analysis and prevention, 50, 1096-103.

Horrey, W. J., \& Wickens, C. D. (2004). Driving and Side Task Performance: The Effects of Display Clutter, Separation, and Modality. Human Factors: The Journal of the Human Factors and Ergonomics Society, 46(4), 611624.

Jamson, A. H., \& Merat, N. (2005). Surrogate invehicle information systems and driver behaviour: Effects of visual and cognitive load in simulated rural driving. Transportation Research Part F: Traffic Psychology and Behaviour, 8(2), 79-96.

Klauer, S., Klauer, S., Dingus, T. a., Dingus, T. a., Neale, V. L., Neale, V. L., ... Ramsey, D. (2006). The Impact of Driver Inattention On Near Crash/Crash Risk: An Analysis Using the 100-Car Naturalistic Driving Study Data. Analysis(April), 226.

Kountouriotis, G. K., Wilkie, R. M., Gardner, P. H., \& Merat, N. (2015). Looking and thinking when driving: The impact of gaze and cognitive load on steering. Transportation Research Part F: Traffic Psychology and Behaviour, 34, 108-121.

Kubose, T. T., Bock, K., Dell, G. S., Garnsey, S. M., Kramer, A. F., \& Mayhugh, J. (2006). The effects of speech production and speech comprehension on simulated driving performance. Applied Cognitive Psychology, 20(1), 43-63.

Lamble, D., Kauranen, T., Laakso, M., \& Summala, H. (1999). Cognitive load and detection thresholds in car following situations: safety implications for using mobile (cellular) telephones while driving. Accident Analysis \& Prevention, 31(6), 617-623.

Land, M., \& Horwood, J. (1995). Which parts of the road guide steering? Nature, 377(6547), 339-340.

Lee, Y.-C., Lee, J. D., \& Ng Boyle, L. (2007). Visual Attention in Driving: The Effects of Cognitive Load and Visual Disruption. Human Factors: The Journal of the Human Factors and Ergonomics Society, 49(4), 721-733.

Liang, Y., \& Lee, J. D. (2010). Combining cognitive and visual distraction: less than the sum of its parts. Accident; analysis and prevention, 42(3), 881-90.

Macdonald, W. A., \& Hoffmann, E. R. (1980). Review of relationships between steering wheel reversal rate and driving task demand (Vol. 22) (No. 6).

Medeiros-Ward, N., Cooper, J. M., \& Strayer, D. L. (2014). Hierarchical control and driving. Journal of experimental psychology. General, 143(3), 953-8.

Merat, N., \& Jamson, A. H. (2008). The Effect of Stimulus Modality on Signal Detection: Implications for Assessing the Safety of In-Vehicle Technology. Human Factors: The Journal of the Human Factors and Ergonomics Society, 50(1), 145-158.

Merat, N., Kountouriotis, G. K., Tomlinson, A., \& Carsten, O. M. J. (2015). Visual and nonvisual distractions: Are they all that different? To appear in the Proceedings of the Fourth International Conference on Driver Distraction and Inattention, Sydney, Australia.

Michon, J. A. (1985). A critical view of driver behavior models: What do we know, what should we do? In L. Evans \& R. C. Schwing (Eds.), Human behavior and traffic safety. New York, NY: Plenum Press. 
Mühlbacher, D., \& Krüger, H. P. (2011). The effect of car-following on lateral guidance during cognitive load: A study conducted in the multi-driver simulation. 2nd International Conference on Driver Distraction and Inattention (DDI 2011), Göteborg/Schweden.

Recarte, M. A., \& Nunes, L. M. (2000). Effects of verbal and spatial-imagery tasks on eye fixations while driving. Journal of Experimental Psychology: Applied, 6(1), 31-43.

Recarte, M. A., \& Nunes, L. M. (2003). Mental workload while driving: Effects on visual search, discrimination, and decision making. Journal of Experimental Psychology: Applied, 9(2), 119-137.

Reimer, B. (2009). Impact of Cognitive Task Complexity on Drivers' Visual Tunneling. Transportation Research Record: Journal of the Transportation Research Board, 2138(2138), 13-19.

Reimer, B., Mehler, B., Wang, Y., \& Coughlin, J. F. (2010). The impact of systematic variation of cognitive demand on drivers' visual attention across multiple age groups. In Proceedings of the human factors and ergonomics society (Vol. 3, pp. 2052-2056).

Reyes, M. L., \& Lee, J. D. (2008). Effects of cognitive load presence and duration on driver eye movements and event detection performance. Transportation Research Part F: Traffic Psychology and Behaviour, 11(6), 391402.

Salvucci, D. D. (2001). Predicting the effects of incar interface use on driver performance: an integrated model approach. International Journal of Human-Computer Studies, 55(1), 85-107.

Salvucci, D. D., \& Beltowska, J. (2008). Effects of Memory Rehearsal on Driver Performance: Experiment and Theoretical Account. $\mathrm{Hu}$ man Factors: The Journal of the Human Factors and Ergonomics Society, 50(5), 834-844.
Salvucci, D. D., \& Gray, R. (2004). A two-point visual control model of steering. Perception, 33(10), 1233-1248.

Santos, J., Merat, N., Mouta, S., Brookhuis, K., \& de Waard, D. (2005). The interaction between driving and in-vehicle information systems: Comparison of results from laboratory, simulator and real-world studies. Transportation Research Part F: Traffic Psychology and Behaviour, 8(2), 135-146.

Strayer, D. L., \& Johnston, W. A. (2001). Driven to distraction: Dual-Task Studies of Simulated Driving and Conversing on a Cellular Telephone. Psychological Science, 12(6), 462466.

Victor, T. W., Harbluk, J. L., \& Engström, J. A. (2005). Sensitivity of eye-movement measures to in-vehicle task difficulty. Transportation Research Part F: Traffic Psychology and Behaviour, 8(2), 167-190.

Wang, Y.-X., Chuang, C.-N., Chang, C.-L., Wu, Y.-T., Chang, C.-Y., \& Leung, M.k. (2014). Post-functionalized poly(Nvinylcarbazole)s as effective low turn-onvoltage phosphorescent-hosts in polymeric light emitting devices. European Polymer Journal, 52(1), 227-237.

Wickens, C. D. (2002). Multiple resources and performance prediction. Theoretical Issues in Ergonomics Science, 3(2), 159-177.

Wilkie, R. M., \& Wann, J. (2003). Controlling steering and judging heading: Retinal flow, visual direction, and extraretinal information. Journal of Experimental Psychology: Human Perception and Performance, 29(2), 363-378.

Wilkie, R. M., Wann, J. P., \& Allison, R. S. (2008). Active gaze, visual look-ahead, and locomotor control. Journal of experimental psychology. Human perception and performance, 34(5), 1150-64. 